\title{
Bioelectrical impedance analysis of body composition: influence of a newly implanted cardiac device
}

\author{
Luiz Wellington Pinto, Silvia Veloso Gandra, Matheus de Carvalho Alves, Isabel Gomes, Eduardo Back Sternick
}

Post Graduate Department, Faculdade Ciências Médicas - MG, Belo Horizonte, Brazil

E-mail any correspondence to: eduardosternick@cienciasmedicasmg.edu.br

\begin{abstract}
Current guidelines do not recommend bioelectrical impedance analysis (BIA) in patients with implanted cardiac devices. There is no data on the influence of such devices over the parameters assessed by BIA. We aimed to assess the influence of cardiac devices on the parameters assessed by BIA as well as to evaluate the likelihood of electromagnetic interference of BIA in patients with implanted cardiac devices. Sixty-two consecutive patients over 18 years of age who underwent single (PM) or multisite (CRT) pacemaker or defibrillator (ICD) implantation were included. Body composition assessment was done using a single frequency device, on both right and left sides, before and after cardiac device implantation. During BIA analysis after device implantation, we did real-time telemetry to assess electromagnetic interference. Patients were $67+14$ years old and $51.6 \%$ male. PM was implanted in 52 patients (83.9\%), ICD in 7 (11.3\%), ICD with CRT in 2 (3.2\%) and CRT in 1 (1.6\%). During real-time telemetry, there was no electromagnetic interference including interruption of telemetry. Default device programming did not change after BIA assessment. After surgery, resistance and fat mass were smaller, while cellular mass, fat-free mass, metabolic rate and total body water/ body weight increased, on right and left sides measurements. We concluded that decreased resistance and related parameters after device implantation were probably influenced to a change in hydration status, regardless of the implanted device. Bioimpedance analysis is safe in patients with an implanted cardiac device.
\end{abstract}

Keywords: Bioimpedance, pacemaker, cardioverter-defibrillator, ICD, electromagnetic interference

\section{Introduction}

Bioelectrical impedance analysis (BIA) of body composition measures resistance and reactance as well as other parameters calculated by mathematical equations. A variety of methods are used for BIA analysis, including skin-fold measurements, single, multifrequency, and segmental BIA, bioimpedance spectroscopy, and more complex methods like dual energy X-ray absorptiometry [1, 2].

BIA assessment has gained widespread popularity because it is easy to perform, portable, inexpensive, observer independent, and safe. Current indication has expanded well beyond clinical research in physiologic and pathologic conditions, including obesity [3], nutritional assessment in training programs of elite athletes [4], nutritional evaluation [5], and prognostic estimates of patients with AIDS [6], cancer [7-9], hyperthyroidism [10], heart failure [11], hemodialysis [12], chronic liver disease [13], wound healing [14], and more recently used in the follow-up of patients with neuromuscular disorders [15].

$\mathrm{BIA}$ is not recommended in patients with an electronic cardiac device, like pacemaker (PM), implantable cardiac defibrillator (ICD), and resynchronizers (CRT). There is a single small study where electromagnetic interference was not detected while using BIA in patients with ICD [16]. In addition, there is no data on the influence of implanted cardiac devices on the parameters of body composition as measured by BIA. The aim of this study is to assess the influence of a newly implanted cardiac device on BIA parameters as well as to detect any electromagnetic interference caused by the electrical current used during BIA measurements on pacemakers and defibrillators. 
Materials and methods

Population

Sixty-two consecutive patients over 18 years old with an indication for pacemaker or defibrillator implantation were included in the study, after signing an informed consent. Exclusion criteria included decompensated heart failure (New York Heart Association - NYHA functional classes III and IV) or any edema clinically detected, and previously implanted cardiac device. The study population comprised 62 patients with a mean age of $67 \pm 14$ years (range of 19 to 91 ), 32 males (51.6\%) with a mean body mass index (BMI) of $25.5+4.2$ $\mathrm{kg} / \mathrm{m}^{2}$ (table 1).

\section{Body composition assessment}

Measurements were taken in the morning after a fast of 8 hour (patients were told to drink one glass with $200 \mathrm{ml}$ of water 4 hours before the procedure). Diuretics were discontinued 24 hours before surgery. Patients were measured and weighed naked, with a metric medical scale with a height rod, before surgery. Results were approximated to the nearest $0.1 \mathrm{~kg}$, and height to the nearest $0.5 \mathrm{~cm}$. Bioimpedance analysis was done in a supine position, with a BIA 450 (Biodynamics ${ }^{\mathrm{TM}}$, Shoreline, WA, USA), a portable, single sine waveform $50 \mathrm{kHz}$ frequency device, and 0.8 milliamperes of alternating current. Connection to each patient was done using 8 dischargeable Conmed ${ }^{\mathrm{TM}}$ electrodes, model Heartbeat (standard tetrapolar wrist/ankle arrangement). Bioimpedance was measured as usual in the right side, and also from the left due to the fact that all cardiac devices were implanted at the left subclavicular region. Measured parameters included resistance $(R)$, reactance $(X)$, phase angle $(\alpha)$, body capacitance (C) fat-free mass (FFM), body cellular mass (BCM), extracellular mass (ECM), fat mass (FM), ECM/BCM, body mass index (BMI), total body water (TBW), intracellular water (ICW), extracellular water (ECW), TBW/FFM, TBW/body weight and a print-out was obtained from each measurement.

\section{Implanted devices and telemetry}

Cardiac devices were either Saint Jude Medical ${ }^{\mathrm{TM}}$ or Biotronik $^{\mathrm{TM}}$, and were listed in table 1. During post implant BIA measurements, we undertook real-time telemetry using Biotronik $^{\mathrm{TM}}$ ICS3000, or Saint Jude Medical ${ }^{\mathrm{TM}}$ Merlin 3650 programmers. ICDs were disabled during BIA measurements during real-time telemetry to avoid risk of inappropriate shocks.

\section{Statistical analysis}

Categorical variables were presented as percentages and counts, and continuous variables as mean \pm standard deviation (SD). Variables measured in $\mathrm{kg}$ were assessed as percentages of total body weight. The normality of the variables was verified by the Shapiro-Wilk test. Comparison of the means of the parameters measured by bioimpedance, were assessed by Student's $t$-test for independent and paired samples.
Association between categorical variables were assessed using the chi-square, or Fisher's exact test. We used the freesoftware $R$ version 3.1.3 with a significance level of $5 \%$.

\begin{tabular}{|c|c|}
\hline Variables & Statistics \\
\hline \multicolumn{2}{|l|}{ Patients } \\
\hline \multicolumn{2}{|l|}{ Gender } \\
\hline Female & $30(48.4 \%)$ \\
\hline Male & 32 (51.6\%) \\
\hline Age (years) & $67.3 \pm 14.7$ \\
\hline BMI $\left(\mathrm{kg} / \mathrm{m}^{2}\right)$ & $25.5 \pm 4.2$ \\
\hline Time of surgery (minutes) & $60.5 \pm 14.2$ \\
\hline Amount of fluids during surgery $(\mathrm{ml})$ & $487.9 \pm 223.4$ \\
\hline \multicolumn{2}{|l|}{ Medications } \\
\hline Mean number & $3.6 \pm 1.8$ \\
\hline Diuretics & $30(49.2 \%)$ \\
\hline Glucocorticosteroids & $2(3.3 \%)$ \\
\hline \multicolumn{2}{|l|}{ Implanted devices } \\
\hline \multicolumn{2}{|l|}{ Devices } \\
\hline PM & $52(83.9 \%)$ \\
\hline ICD & $7(11.3 \%)$ \\
\hline $\mathrm{ICD}+\mathrm{CRT}$ & $2(3.2 \%)$ \\
\hline CRT & $1(1.6 \%)$ \\
\hline \multicolumn{2}{|l|}{ Generators } \\
\hline Etrinsa 8DR-T & $31(50 \%)$ \\
\hline Endurity DR & $15(24.2 \%)$ \\
\hline Ilesto 5DR-T & $6(9.7 \%)$ \\
\hline Unify Quadra CRT-D & $3(4.8 \%)$ \\
\hline Others* & $7(11.3 \%)$ \\
\hline \multicolumn{2}{|l|}{ RA leads } \\
\hline Safio S53 & $36(60 \%)$ \\
\hline Tendril STS 2088TC-58 & $14(23.3 \%)$ \\
\hline Optisense 1999-52 & $4(6.7 \%)$ \\
\hline Others ${ }^{* *}$ & $6(10 \%)$ \\
\hline \multicolumn{2}{|l|}{ RV leads } \\
\hline $\mathrm{S} 60$ & $36(58.1 \%)$ \\
\hline Tendril STS 2088TC-58 & $12(19.4 \%)$ \\
\hline Protego DS4 & $3(4.8 \%)$ \\
\hline Others ${ }^{* * *}$ & $11(17.7 \%)$ \\
\hline \multicolumn{2}{|l|}{ LV leads } \\
\hline Quartet 1458Q-86 & $2(66.7 \%)$ \\
\hline Quickflex 1258T-86 & $1(33.3 \%)$ \\
\hline Pro-MRI & $42(67.7 \%)$ \\
\hline
\end{tabular}

Notes: In the category "others" were included generator or cables with less than 3 units: *Accent $D R$, Allure Quadra RF, Endurity $D R$ MRI, Endurity VR MRI, Entovis. ** Isoflex 1948-58, Promri SD 65/16, Solia S53, *** Durata 7120Q-58, Durata 7120Q-60, Optisense 199952, Tendril 1999-58, Tendril LPA1200M-58, Tendril MRI LPA 1200M58, Tendril ST Optim 1888-TC Pro-MRI - compatible with magnetic resonance imaging.

Results

Indications for cardiac device were atrioventricular block in 38 patients $(61.3 \%)$, atrial fibrillation with high degree atrioventricular block in 7 (11.3\%), sick sinus syndrome in 6 (9.7\%), atrioventricular node ablation in $1(1.6 \%)$, sustained monomorphic ventricular tachycardia in 9 (14.5\%), and left bundle branch block and heart failure in 1 (1.6\%). Fourteen patients (22.6\%) did not have structural heart disease, while 16 (25.8\%) had ischemic cardiomyopathy, 12 (19.4\%) had hypertensive cardiomyopathy, 10 (16.1\%) had chagasic cardiomyopathy, 4 (6.5\%) had idiopathic dilated cardiomyopathy, $4(6.4 \%)$ had rheumatic heart disease (3 
mitral valve, and 1 aortic valve bioprosthesis), and 2 (3.2\%) had hypertrophic cardiomyopathy.

Most important associated morbidities were hypertension in 38 patients $(61.3 \%)$, diabetes in $10(16.1 \%)$, and

hypothyroidism in $6(9.7 \%)$.

Patients were taking $3.6 \pm 1.8$ medications, and 30 patients (49.2\%) were taking diuretics.

\section{Cardiac devices}

A pacemaker (PM) was implanted in 52 patients (83.9\%), an ICD in 7 (11.3\%), an ICD with CRT in 2 (3.2\%), and CRT in 1

\section{(1.6\%) (table 1)}

Device implantation procedure was performed under light sedation, and lasted in average $60.4 \pm 14.1$ minutes. The average amount of intravenous fluids received during the procedure was $487 \pm 223 \mathrm{ml}$. Only 1 out of the 3 CRT required an epicardial implantation under short-lived general anesthesia. The average time between the end of surgery and post implant BIA measurements was $4.3 \pm 1.1$ minutes.

\section{Bioimpedance parameters}

All impedance variables were normally distributed.

Bioimpedance measurements did not differ as comparing right and left sides, before and after device implantation (table 2). However, after implant, we observed changes in some parameters, as compared with pre-implantation measurements (table 3 ): higher values- body cellular mass $(p<0.02 / p=0.007)$, fat-free mass $(p<0.001 / p<0.001)$, and TBW/ body weight $(p=0.006 / p=0.03)$, and Capacitance, which increased only in the left side assessment (NS[R]/p=0.01[L]); reduced values- Resistance $(p<0.02 / p<0.02)$, and fat mass $(p<0.001 / p<0.001)$, on both sides.

\section{Subgroups analysis}

We analyzed bioimpedance parameters before and after cardiac device implantation, from right and left sides according to the amount of intravenous fluids received during surgery, age, gender, BMI, left ventricular ejection fraction (LVEF), duration of the surgical procedure, type of cardiac device implanted, compatibility to magnetic resonance imaging, and use of diuretics.

\section{Amount of intravenous fluids}

We found that in those patients who received more than 500 $\mathrm{ml}$ there was an increase in body capacitance $(\mathrm{p}=0.04)$, and TBW ( $p=0.03$ ) over the left side (table 4).

\section{Age}

We compared 34 patients (54.8\%) with $>65$ years with 28 (45.2\%) aged $<65$ years. Bioimpedance parameters did not differ when assessing both sides before and after device implantation. However, when comparing the older with the younger patients, we found that before device implantation there were a significant increase in $\mathrm{FM}$, as well as a reduction of $\alpha, C, B C M, F F M, I C W$, and TBW, on both sides. After surgery $\alpha, C$, and ICW lost statistical significance. There was a trend for an increase in resistance before surgery in older patients without statistical significance $(0.05<p<0.10)$, and that trend was less clear after device implantation.

\section{Gender}

Measures among male gender, before and after device implantation, yielded significant smaller values of resistance and FM, and significant larger values of BCM, FFM, ICW, ECW, TBW, and rate between BW/body weight. Capacitance and phase angle were only increased before surgery. Increased $C$ was observed on both sides, but increased phase angle only on the right-side measurement.

\section{$B M I$}

Nine patients (14.5\%) had a BMI $>30 \mathrm{~kg} / \mathrm{m}^{2}$. Those patients had on either side, before and after device implantation, a significantly smaller $\mathrm{R}$, and an increase in TBW.

There were no differences in bioimpedance measures when comparing data before and after surgery when assessing LVEF (< or $>50 \%$ ), duration of the procedure ( $<$ and $>60$ minutes), type of device implanted (ICD vs PM), MRIcompatible vs not compatible devices, patients taking vs those not taking diuretics.

\section{Discussion}

The main findings of our study were a significant change in a number of parameters of bioimpedance after device implantation, regardless of the type of the device, pacemaker or ICD, and absence of electromagnetic interference of the alternate current delivered by the BIA 450 on cardiac devices.

\section{Changes in bioimpedance measurements after implant} The major parameters measured from a single frequency BIA are resistance, and reactance, which are used to calculate phase angle. The other parameters are obtained by regression equations, and have limited accuracy to track longitudinal changes, particularly in FM and FFM [17].

Implanted metallic cardiac devices would theoretically not lead to a change in resistance, due to its small volume. The finding of reduction of resistance consistently on both sides, speaks against the influence of implanted cardiac devices. A possible explanation lay in the amount of fluids received during a short time-frame, in patients fasting for 8 hours. Phase angle and reactance did not change after implantation of the cardiac device. Resistance is a component of the equations that yield all other changed parameters, which change because of the change in resistance measures, and can be interpreted as a false positive result.

\section{Real-time telemetry}

During real-time telemetry, there was no oversensing, undersensing, reversion to noise mode, change in default programming, or disruption of telemetry. ICDs were disabled during BIA measurements with real-time telemetry to avoid inappropriate shocks. 
Table 2: Comparison of bioimpedance parameters by body sides before and after cardiac device implantation

\begin{tabular}{|c|c|c|c|c|c|c|}
\hline \multirow{2}{*}{ BIA parameters } & \multicolumn{3}{|c|}{ Pre implantation } & \multicolumn{3}{|c|}{ Post implantation } \\
\hline & Right side & Left side & $P$ value & Right side & Left side & $P$ value \\
\hline Phase angle (degree) & $6.5 \pm 1.4$ & $6.4 \pm 1.5$ & NS & $6.7 \pm 2.3$ & $6.8 \pm 2.2$ & NS \\
\hline Body capacitance (Pf) & $690.7 \pm 257.8$ & $677.2 \pm 214.6$ & NS & $750.4 \pm 354.8$ & $798 \pm 421.6$ & NS \\
\hline Resistance (ohms) & $535.8 \pm 123,6$ & $532,6 \pm 122$ & NS & $514 \pm 101.8$ & $510 \pm 117.5$ & NS \\
\hline Reactance (ohms) & $66.8 \pm 49.5$ & $66.5 \pm 50$ & NS & $59.2 \pm 18.4$ & $59.4 \pm 17.7$ & NS \\
\hline Body cellular mass \% & $31.8 \pm 7.3$ & $31.5 \pm 6.5$ & NS & $33.8 \pm 9.1$ & $33.8 \pm 8$ & NS \\
\hline Extracellular mass \% & $37.1 \pm 5.6$ & $37.5 \pm 5.9$ & NS & $37.6 \pm 7.1$ & $37.7 \pm 7.2$ & NS \\
\hline Fat-free mass \% (FFT) & $68.9 \pm 10.8$ & $69 \pm 10.4$ & NS & $71.4 \pm 9.9$ & $71.5 \pm 10$ & NS \\
\hline Fat mass $\%$ & $30.5 \pm 9.6$ & $29.8 \pm 9.4$ & NS & $27.3 \pm 9$ & $27.2 \pm 9.3$ & NS \\
\hline Intracellular water \% & $52.6 \pm 9.2$ & $52.2 \pm 9.1$ & NS & $53 \pm 9.9$ & $53.6 \pm 9.4$ & NS \\
\hline Extracellular water \% & $44.7 \pm 8.7$ & $45.6 \pm 8.5$ & NS & $44.8 \pm 9.3$ & $44.4 \pm 10.2$ & NS \\
\hline Total body water \% (TBW) & $36 \pm 10.2$ & $36.1 \pm 10$ & NS & $36.4 \pm 9.1$ & $36.9 \pm 9.6$ & NS \\
\hline TBW / FFT & $73.3 \pm 3.2$ & $73 \pm 3.5$ & NS & $73.4 \pm 2.6$ & $73 \pm 6.4$ & NS \\
\hline TBW / Body weight & $50.5 \pm 8.4$ & $50.5 \pm 8.5$ & NS & $52.9 \pm 6.2$ & $54 \pm 7.7$ & NS \\
\hline
\end{tabular}

Notes: $p$-value refers to Student $t$-test for paired samples (NS = non-significant)

Table 3: Comparison of bioimpedance parameters before and after cardiac device implantation on both sides

\begin{tabular}{|c|c|c|c|c|c|c|}
\hline \multirow{2}{*}{ BIA parameters } & \multicolumn{3}{|l|}{$\underline{\text { Right side }}$} & \multicolumn{3}{|l|}{ Left side } \\
\hline & Pre implantation & Post implantation & $P$ value & Pre implantation & Post implantation & P value \\
\hline Phase angle (degree) & $6.5 \pm 1.4$ & $6.7 \pm 2.3$ & NS & $6.4 \pm 1.5$ & $6.8 \pm 2.2$ & NS \\
\hline Body capacitance (Pf) & $690.7 \pm 257.8$ & $750.4 \pm 354.8$ & NS & $677.2 \pm 214.6$ & $798 \pm 421.6$ & 0.013 \\
\hline Resistance (ohms) & $535.8 \pm 123.6$ & $514 \pm 101.8$ & 0.018 & $532.6 \pm 122$ & $510.3 \pm 117.5$ & 0.024 \\
\hline Reactance (ohms) & $66.8 \pm 49.5$ & $59.2 \pm 18.4$ & NS & $66.5 \pm 50$ & $59.4 \pm 17.7$ & NS \\
\hline Body cellular mass (\%) & $31.8 \pm 7.3$ & $33.8 \pm 9.1$ & 0.027 & $31.5 \pm 6.5$ & $33.8 \pm 8$ & 0.007 \\
\hline Extracellular mass (\%) & $37.1 \pm 5.6$ & $37.6 \pm 7.1$ & NS & $37.5 \pm 5.9$ & $37.7 \pm 7.2$ & NS \\
\hline Fat-free mass (\%) (FFT) & $68.9 \pm 10.8$ & $71.4 \pm 9.9$ & $<0.001$ & $69 \pm 10.4$ & $71.5 \pm 10$ & $<0.001$ \\
\hline Fat mass (\%) & $30.5 \pm 9.6$ & $27.3 \pm 9$ & $<0.001$ & $29.8 \pm 9.4$ & $27.2 \pm 9.3$ & $<0.001$ \\
\hline Intracellular water (\%) & $52.6 \pm 9.2$ & $53 \pm 9.9$ & NS & $52.2 \pm 9.1$ & $53.6 \pm 9.4$ & NS \\
\hline Extracellular water (\%) & $44.7 \pm 8.7$ & $44.8 \pm 9.3$ & NS & $45.6 \pm 8.5$ & $44.4 \pm 10.2$ & NS \\
\hline Total body water (\%) (TBW) & $36 \pm 10.2$ & $36.4 \pm 9.1$ & NS & $36.1 \pm 10$ & $36.9 \pm 9.6$ & NS \\
\hline TBW / FFT & $73.3 \pm 3.2$ & $73.4 \pm 2.6$ & NS & $73 \pm 3.5$ & $73 \pm 6.4$ & NS \\
\hline TBW / Body weight & $50.5 \pm 8.4$ & $52.9 \pm 6.2$ & 0.006 & $50.5 \pm 8.5$ & $54 \pm 7.7$ & 0.003 \\
\hline
\end{tabular}

\begin{tabular}{|c|c|c|c|}
\hline BIA parameters & $\begin{array}{l}<500 \mathrm{ml} \\
(n=23)\end{array}$ & $\begin{array}{l}\geq 500 \mathrm{ml} \\
(n=39)\end{array}$ & $P$ value \\
\hline Phase angle (degree) & $6.3 \pm 1.8$ & $7.5 \pm 2.5$ & NS \\
\hline Body capacitance (Pf) & $681.4 \pm 255.2$ & $949.5 \pm 509.3$ & 0.04 \\
\hline Resistance (ohms) & $531.7 \pm 107.3$ & $478.4 \pm 125.8$ & NS \\
\hline Reactance (ohms) & $58.2 \pm 17.5$ & $60.6 \pm 19.3$ & NS \\
\hline Body cellular mass (\%) & $31.4 \pm 7.3$ & $35.3 \pm 7.7$ & NS \\
\hline Extracellular mass (\%) & $37.9 \pm 7.8$ & $36.6 \pm 7.2$ & NS \\
\hline Fat-free mass (\%) (FFT) & $69.3 \pm 12.4$ & $71.7 \pm 9.4$ & NS \\
\hline Fat mass (\%) & $28.2 \pm 11.1$ & $26.8 \pm 9.3$ & NS \\
\hline Intracellular water (\%) & $50.9 \pm 10.6$ & $55.3 \pm 7.9$ & NS \\
\hline Extracellular water (\%) & $45.4 \pm 10.4$ & $41.9 \pm 10.8$ & NS \\
\hline Total body water (\%) (TBW) & $34.1 \pm 8.8$ & $40.9 \pm 9.8$ & 0.02 \\
\hline TBW / FFT & $74 \pm 3.4$ & $71.6 \pm 10.7$ & NS \\
\hline TBW / Body weight & $52.6 \pm 7.7$ & $55.8 \pm 9.4$ & NS \\
\hline
\end{tabular}

\section{Low likelihood of a cardiac device role on changes of BIA parameters}

It is known that the geometry and size of different body segments plays a major role on the results of bioimpedance measurements. The smaller the cross-section the larger the impact, particularly because of a disproportionately higher resistance to the electrical current. The upper and lower limbs have a much smaller cross-sectional area and volume, as compared to the trunk. The region of the trunk represents $\sim 50 \%$ of the body fat-free mass but only $\sim 10 \%$ of body resistance. In contrast, one arm and one leg comprise $\sim 25 \%$ of the fat-free mass and up to $90 \%$ of total body resistance $[18,19]$. Those proportions can be explained by virtue of the nature of the regression equations used in bioimpedance analysis, where body parts with smaller crosssection areas make a dominant effect on resistance. This suggests a lack of influence of the implanted cardiac devices and leads on bioimpedance parameters because of the small volume of the device generator, and leads, in a body 
segment having a much larger cross-sectional area and volume.

\section{Subgroup analysis}

Influence of older age, and obesity, observed in the present study, reflects the expected variability caused by different content of TBW, FFM, and FM, as assessed before device implantation [20]. However, in the older patients ( $>65$ years old), some parameters like $\alpha, C, I C W$, lost statistical significance after surgery, while reduction of BCM, FFM, and TBW, and increase in FM were detected before and after surgery in older patients. We hypothesize that rehydration while decreasing resistance also changed the other parameters, which were computed using Resistance in their regression equations. In spite of not achieving statistical significance there was a trend for increase $\mathrm{R}$ in older patients before surgery ( $10 \%$ larger $\mathrm{R}$ in older patients), which was lost after surgery.

Regarding male gender, rehydration probably contributed to post-surgical change in bioimpedance parameters, particularly not finding a higher $\alpha$, which is expected in males, and was found before surgery [13]. We did not detect any difference in bioimpedance parameters, as comparing patients with normal and reduced LVEF. By protocol, patients with congestive heart failure were excluded from the study. In spite of a low mean LVEF $(39 \pm 9 \%)$ in the group of reduced LVEF, the patients were in NYHA functional class I or II, without volume overload.

There was an increase in C and TBW in patients who received larger amount of fluids before the post- implant bioimpedance measurements (table 4). Those findings are consistent with the role of hydration status as the cause of the changes in parameters found after the surgical procedure. Interestingly, there was also a significant change in FFM $(p<0,001)$. The assumption of a constant hydration is the basis for FFM estimates with single frequency BIA.

Overestimation of FFM has been reported with expansion of extracellular volume [18]. It is worth noting that a relatively small amount of fluids ( $<500 \mathrm{ml}$ ) caused such a significant reduction in resistance, maybe the key issue was the relatively short period of time ( $<65$ minutes) of fluids administration and post implantation BIA measurements.

\section{Lack of electromagnetic interference on pacemaker and ICD}

The alternate current delivered by the BIA 450 lasts less than 1 second, and is not long enough to be detected by ICDs. There was not a single instance of oversensing, change in default programming, or disruption of telemetry. Our results were consistent with the ones reported by Buch et al [16].

\section{Limitations of the study}

The patients were not weighted before post-surgery bioimpedance assessment. However, as the amount of fluids was in average approximately $500 \mathrm{ml}$, total body weight would not have been significantly different, once results were approximated to the nearest $0.1 \mathrm{~kg}$.

\section{Conclusion}

Single frequency bioimpedance measurements of body composition changed after device implantation in the thorax. Decreased resistance and related parameters are probably related to a change in hydration status. Bioimpedance analysis is safe in patients with implanted cardiac devices.

\section{References}

1. Jaffrin MY, Morel H. Body fluid volumes measurements by impedance: a review of bioimpedance spectroscopy (BIS) and bioimpedance analysis (BIA) methods. Med Eng Phys. 2008;30:1257-69. https://doi.org/10.1016/j.medengphy.2008.06.009

2. Boneva-Asiova Z, Boyanov MA. Body composition analysis by leg-to-leg bioelectrical impedance and dual-energy X-ray absorptiometry in non-obese and obese individuals. Diabetes Obes Meta. 2008;10:1012-8. https://doi.org/10.1111/j.1463-1326.2008.00851.x

3. Johnstone AM, Faber P, Gibney ER, Lobley GE, Stubbs RJ, Siervo $M$. Measurement of body composition changes during weight loss in obese men using multi-frequency bioelectrical impedance analysis and multicompartment models. Obes Res Clin Pract. 2014;8:e46-e54. https://doi.org/10.1016/j.orcp.2012.07.006

4. Matias CN, Santos DA, Fields DA, Sardinha LB, Silva AM. Is bioelectrical impedance spectroscopy accurate in estimating changes in fat-free mass in judo athletes? J Sports Sci. 2012;30:1225-33. https://doi.org/10.1080/02640414.2012.692481

5. Bell SJ, Bistrian BR, Connolly CA, Forse RA. Body composition changes in patients with human immunodeficiency virus infection. Nutrition. 1997;13:629-32. https://doi.org/10.1016/S0899-9007(97)83004-3

6. Ott $\mathrm{M}$, Fischer $\mathrm{H}$, Polat $\mathrm{H}$, Helm EB, Frenz $\mathrm{M}$, Caspary WF, et al. Bioelectrical impedance analysis as a predictor of survival in patients with human immunodeficiency virus infection. J Acquir Immune Defic Syndr Hum Retrovirol. 1995;9:20-5. https://doi.org/10.1097/00042560-199505010-00003

7. Gupta D, Lammersfeld CA, Vashi PG, King J, Dahlk SL, Grutsch $\mathrm{JF}$, et al. Bioelectrical impedance phase angle as a prognostic indicator in breast cancer. BMC Cancer. 2008;8:249-66. https://doi.org/10.1186/1471-2407-8-249

8. Gupta D, Lis CG, Dahlk SL, King J, Vashi PG, Grutsch JF, et al. The relationship between bioelectrical impedance phase angle and subjective global assessment in advanced colorectal cancer. Nutr J. 2008;7(1):7-19. https://doi.org/10.1186/1475-2891-7-19

9. Gupta D, Lammersfeld CA, Vashi PG, King J, Dahlk SL, Grutsch $\mathrm{JF}$, et al. Bioelectrical impedance phase angle in clinical practice: implications for prognosis in stage IIIB and IV nonsmall cell lung cancer. BMC Cancer. 2009;9:37-43. https://doi.org/10.1186/1471-2407-9-37 
10. Seppel T, Kosel A, Schlaghecke R. Bioelectrical impedance assessment of body composition in thyroid disease. Eur J Endocrinol. 1997;136:493-8.

https://doi.org/10.1530/eje.0.1360493

11. Tang WHW, Tong W. Measuring impedance in congestive heart failure: current options and clinical applications. Am Heart J. 2009;157:402-11. https://doi.org/10.1016/j.ahj.2008.10.016

12. Maggiore $Q$, Nigrelli S, Ciccarelli C, Grimaldi C, Rossi GA, Michelassi C. Nutritional and prognostic correlates of bioimpedance indexes in hemodialysis patients. Kidney Int. 1996;50(6):2103-8. https://doi.org/10.1038/ki.1996.535

13. Selberg O, Selberg D. Norms and correlates of bioimpedance phase angle in healthy human subjects hospitalized patients, and patients with liver cirrhosis. Eur J Appl Physiol. 2002;86:509-16. https://doi.org/10.1007/s00421-001-0570-4

14. Lukaski HC, Moore M. Bioelectrical impedance assessment of wound healing. J Diabetes Sci Technol. 2012;6:209-12. https://doi.org/10.1177/193229681200600126

15. Rutkove SB. Electrical impedance myography: background, current state, and future directions. Muscle Nerve. 2009;40:936-46. https://doi.org/10.1002/mus.21362
16. Buch E, Bradfield J, Larson T, Horvich T. Effect of bioimpedance body composition analysis on function of implanted cardiac devices. Pacing Clin Electrophysiol 2012;35(6):681-4. https://doi.org/10.1111/j.1540-8159.2012.03377.x

17. Moon JR. Body composition in athletes and sports nutrition: an examination of the bioimpedance analysis technique. Eur J Clin Nutr. 2013;67(Suppl 1):S54-9. https://doi.org/10.1038/ejcn.2012.165

18. Mulasi U, Kuchnia AJ, Cole AJ, Earthman CP. Bioimpedance at the bedside: current applications, limitations, and opportunities. Nutr Clin Pract. 2015;30:180-93. https://doi.org/10.1177/0884533614568155

19. Shafer KJ, Siders WA, Johnson LK, Lukaski HC. Validity of segmental multiple-frequency bioelectrical impedance analysis to estimate body composition of adults across a range of body mass indexes. Nutrition. 2009;25(1):25-32. https://doi.org/10.1016/j.nut.2008.07.004

20. Dittmar M. Reliability and variablility of bioimpedance measures in normal adults: effects of age, gender, and body mass. Am J Phys Anthropol. 2003;122(4):361-70. https://doi.org/10.1002/ajpa.10301 